\title{
Multilinear Calderón-Zygmund operators on weighted Hardy spaces
}

\author{
by \\ Wenjuan Li (Beijing), Qingying Xue (Beijing) and \\ Kôzô Yabuta (Sanda)
}

\begin{abstract}
Grafakos-Kalton [Collect. Math. 52 (2001)] discussed the boundedness of multilinear Calderón-Zygmund operators on the product of Hardy spaces. Then Lerner et al. [Adv. Math. 220 (2009)] defined $A_{\vec{p}}$ weights and built a theory of weights adapted to multilinear Calderón-Zygmund operators. In this paper, we combine the above results and obtain some estimates for multilinear Calderón-Zygmund operators on weighted Hardy spaces and also obtain a weighted multilinear version of an inequality for multilinear fractional integrals, which is related to the classical Trudinger inequality.
\end{abstract}

1. Introduction. Multilinear Calderón-Zygmund theory is a natural generalization of the linear case. In recent years, many authors have been interested in these topics ([5, 6], 4], 20], [15], 13], 21], 3] and [23]). Multilinear singular integral operators have recently attracted more and more attention. The class of multilinear singular integral operators with standard Calderón-Zygmund kernels provides the starting point of the theory. So we first recall the definition of multilinear Calderón-Zygmund operators.

Definition 1 ([6]). Let $T$ be a multilinear operator initially defined on the $m$-fold product of the Schwartz space and taking values in the space of tempered distributions,

$$
T: \mathscr{S}\left(\mathbb{R}^{n}\right) \times \cdots \times \mathscr{S}\left(\mathbb{R}^{n}\right) \rightarrow \mathscr{S}^{\prime}\left(\mathbb{R}^{n}\right) .
$$

We say that $T$ is an m-linear Calderón-Zygmund operator if for some $1 \leq$ $q_{j}<\infty$, it extends to a bounded multilinear operator from $L^{q_{1}} \times \cdots \times L^{q_{m}}$ to $L^{q}$, where $1 / q=1 / q_{1}+\cdots+1 / q_{m}$, and there exists a function $K$, defined off the diagonal $x=y_{1}=\cdots=y_{m}$ in $\left(\mathbb{R}^{n}\right)^{m+1}$, satisfying

$$
T\left(f_{1}, \ldots, f_{m}\right)(x)=\int_{\left(\mathbb{R}^{n}\right)^{m}} K\left(x, y_{1}, \ldots, y_{m}\right) f_{1}\left(y_{1}\right) \cdots f_{m}\left(y_{m}\right) d y_{1} \cdots d y_{m}
$$

2010 Mathematics Subject Classification: Primary 42B20; Secondary 42B25.

Key words and phrases: multiple weights, weighted norm inequalities, multilinear Calderón-Zygmund operators, weighted Hardy spaces. 
for all $x \notin \bigcap_{j=1}^{m} \operatorname{supp} f_{j}$;

$$
\left|K\left(y_{0}, \ldots, y_{j}, \ldots, y_{m}\right)-K\left(y_{0}, \ldots, y_{j}^{\prime}, \ldots, y_{m}\right)\right| \leq \frac{A\left|y_{j}-y_{j}^{\prime}\right|^{\varepsilon}}{\left(\sum_{k, l=0}^{m}\left|y_{k}-y_{l}\right|\right)^{m n+\varepsilon}},
$$

for some $\varepsilon>0$ and all $0 \leq j \leq m$, whenever $\left|y_{j}-y_{j}^{\prime}\right| \leq \frac{1}{2} \max _{0 \leq k \leq m}\left|y_{j}-y_{k}\right|$.

As in the linear theory, a certain amount of extra smoothness is required for these operators to have such boundedness properties. We will assume that $K\left(y_{0}, y_{1}, \ldots, y_{m}\right)$ satisfies the following estimates:

$$
\left|\partial_{y_{0}}^{\alpha_{0}} \cdots \partial_{y_{m}}^{\alpha_{m}} K\left(y_{0}, y_{1}, \ldots, y_{m}\right)\right| \leq \frac{A_{\alpha}}{\left(\sum_{k, l=0}^{m}\left|y_{k}-y_{l}\right|\right)^{m n+|\alpha|}}
$$

for all $|\alpha| \leq N$, where $\alpha=\left(\alpha_{0}, \ldots, \alpha_{m}\right)$ is an ordered set of $n$-tuples of nonnegative integers, $|\alpha|=\left|\alpha_{0}\right|+\cdots+\left|\alpha_{m}\right|$, where $\left|\alpha_{j}\right|$ is the order of each multiindex $\alpha_{j}$, and $N$ is a large integer to be determined later.

The initial work on these operators was done in [5], 6] by Coifman and Meyer in the 70's. Later on, the topic was taken up by several authors, including Christ and Journé [4, Kenig and Stein [20, and Grafakos and Torres [15]. This last work studied multilinear Calderón-Zygmund theory, including the unweighted estimates of multilinear Calderón-Zygmund operators in the weak and strong sense. Weighted settings were studied systematically in [16]; it was shown that if $T$ is an $m$-linear Calderón-Zygmund operator, $1 / p_{1}+\cdots+1 / p_{m}=1 / p$ and $p_{0}=\min \left\{p_{j}\right\}>1$, then $T$ is bounded from $L_{\omega}^{p_{1}} \times \cdots \times L_{\omega}^{p_{m}}$ into $L_{\omega}^{p}$, provided that the weight $\omega$ is in the class $A_{p_{0}}$.

In 2001, Grafakos and Kalton [13] proved the boundedness of multilinear Calderón-Zygmund operators on products of Hardy spaces. They gave the following result:

Theorem A ([13]). Let $1<q_{1}, \ldots, q_{m}, q<\infty$ be fixed indices satisfying

$$
\frac{1}{q_{1}}+\cdots+\frac{1}{q_{m}}=\frac{1}{q}
$$

and let $0<p_{1}, \ldots, p_{m}, p \leq 1$ be real numbers satisfying

$$
\frac{1}{p_{1}}+\cdots+\frac{1}{p_{m}}=\frac{1}{p} \text {. }
$$

Suppose that $K$ satisfies (1.3) with $N=[n(1 / p-1)]$. Let $T$ be related to $K$ and assume that $T$ admits an extension that maps $L^{q_{1}} \times \cdots \times L^{q_{m}}$ into $L^{q}$ with norm $B$. Then $T$ extends to a bounded operator from $H^{p_{1}} \times \cdots \times H^{p_{m}}$ into $L^{p}$. 
Recently, the theory of weighted multilinear Calderón-Zygmund singular integral operators was established in [21] by Lerner et al. New more refined multiple maximal function

$$
\mathcal{M}(\vec{f})(x)=\sup _{Q \ni x} \prod_{i=1}^{m} \frac{1}{|Q|} \int_{Q}\left|f_{i}\left(y_{i}\right)\right| d y_{i}
$$

was defined and used in [21] to characterize the class of $A_{\vec{p}}$ weights and to obtain some weighted estimates for multilinear Calderón-Zygmund singular integral operators. So let us recall the definition of $A_{\vec{p}}$ weights.

For exponents $p_{1}, \ldots, p_{m}$, we will often write $p$ for the number given by $1 / p=1 / p_{1}+\cdots+1 / p_{m}$, and $\vec{p}$ for the vector $\vec{p}=\left(p_{1}, \ldots, p_{m}\right)$.

Definition 2 ([21]). Let $1 \leq p_{1}, \ldots, p_{m}<\infty$. Given $\vec{\omega}=\left(\omega_{1}, \ldots, \omega_{m}\right)$, set

$$
\nu_{\vec{\omega}}=\prod_{j=1}^{m} \omega_{j}^{p / p_{j}} .
$$

We say that $\vec{\omega}$ satisfies the $A_{\vec{p}}$ condition if

$$
\sup _{Q}\left(\frac{1}{|Q|} \int_{Q} \prod_{i=1}^{m} \omega_{i}^{p / p_{i}}\right)^{1 / p} \prod_{i=1}^{m}\left(\frac{1}{|Q|} \int_{Q} \omega_{i}^{1-p_{i}^{\prime}}\right)^{1 / p_{i}^{\prime}}<\infty .
$$

When $p_{i}=1,\left(|Q|^{-1} \int_{Q} \omega_{i}^{1-p_{i}^{\prime}}\right)^{1 / p_{i}^{\prime}}$ is understood as $\left(\inf _{Q} \omega_{i}\right)^{-1}$.

In particular, when $m=1$, we note that $A_{\vec{p}}$ degenerates to the classical $A_{p}$ weight. Moreover, if $m=1$ and $p_{i}=1$, then this class of weights coincides with the classical $A_{1}$ weights. It is well-known that if $\omega \in A_{p}$ for $1<p<\infty$, then $\omega \in A_{r}$ for all $r>p$ and $\omega \in A_{q}$ for some $1<q<p$. We thus use $q_{\omega}:=\inf \left\{q>1: \omega \in A_{q}\right\}$ to denote the critical index of $\omega$. We will refer to (1.4) as the multilinear $A_{\vec{p}}$ condition.

A result in [21] can be stated as follows.

Theorem B ([21]). Let $T$ be an m-linear Calderón-Zygmund operator, satisfying (1.1), (1.2), $1 / p=1 / p_{1}+\cdots+1 / p_{m}$, let $1<p_{i}<\infty$ and let $\vec{\omega}$ satisfy the $A_{\vec{p}}$ condition. Then

$$
\|T(\vec{f})\|_{L_{\nu_{\vec{\omega}}}^{p}} \leq C \prod_{i=1}^{m}\left\|f_{i}\right\|_{L_{\omega_{i}}^{p_{i}}}
$$

Weighted estimates for a class of multilinear fractional type operators were proved in [3] and [23]. These results together with [21] answered an open problem in [17], concerning the existence of multiple weights for which weighted estimates like (1.5) hold.

On the other hand, in 1989, Strömberg and Torchinsky in [25] defined weighted Hardy spaces and obtained the boundedness of Calderón-Zygmund operators on them. 
We will use García-Cuerva's atomic decomposition theory [9] for weighted Hardy spaces.

Definition 3. Assume that $\omega \in A_{q}$ with critical index $q_{\omega}$. Let $[\cdot]$ be the greatest integer function. For $s \in \mathbb{Z}$ satisfying $s \geq\left[n\left(q_{\omega} / p-1\right)\right]$, a real-valued function $a(x)$ is called a $(p, q, s)$-atom centered at $x_{0}$ with respect to $\omega$ (or an $\omega$ - $(p, q, s)$-atom centered at $\left.x_{0}\right)$ if

(a) $a \in L_{\omega}^{q}\left(\mathbb{R}^{n}\right)$ and is supported in a cube $Q$ centered at $x_{0}$;

(b) $\|a\|_{L_{\omega}^{q}} \leq \omega(Q)^{1 / q-1 / p}$;

(c) $\int_{\mathbb{R}^{n}} a(x) x^{\alpha} d x=0$ for every multi-index $\alpha$ with $|\alpha| \leq s$.

When $q=\infty, L_{\omega}^{\infty}$ will be taken to mean $L^{\infty}$ and $\|f\|_{L_{\omega}^{\infty}}=\|f\|_{\infty}$.

Theorem C ([9]). Let $w \in A_{q}, 0<p \leq 1 \leq q \leq \infty$, and $p \neq q$. For each $f \in H_{\omega}^{p}\left(\mathbb{R}^{n}\right)$, there exist a sequence $a_{i}$ of $\omega-\left(p, q,\left[n\left(q_{w} / p-1\right)\right]\right)$-atoms, and a sequence $\lambda_{i}$ of real numbers with $\sum\left|\lambda_{i}\right|^{p} \leq C\|f\|_{H_{\omega}^{p}}^{p}$ such that $f=\sum \lambda_{i} a_{i}$ both in the sense of distributions and in the $H_{\omega}^{p}$ norm.

In view of Theorems $\mathrm{A}$ and $\mathrm{B}$, it is natural to look for weighted results on multilinear singular integral operators acting on weighted Hardy spaces. The first aim of this paper is to give such results. Our first result is as follows:

Theorem 1.1. Let $1<q_{1}, \ldots, q_{m}, q<\infty$ and $0<p_{1}, \ldots, p_{m}, p \leq 1$ satisfy

$$
\frac{1}{q_{1}}+\cdots+\frac{1}{q_{m}}=\frac{1}{q} \quad \text { and } \quad \frac{1}{p_{1}}+\cdots+\frac{1}{p_{m}}=\frac{1}{p} .
$$

Let $T$ be an m-linear Calderón-Zygmund operator such that $K$ satisfies (1.1)-(1.3) with $N=\max _{1 \leq i \leq m}\left\{\left[n\left(\left(q_{i}\right)_{\omega} / p_{i}-1\right)\right],\left[\left(q_{i} / p_{i}-1\right) m n\right]\right\}$.

(i) If $\omega \in A_{q_{1}} \cap \cdots \cap A_{q_{m}}$, then

$$
\|T(\vec{f})\|_{L_{\omega}^{p}} \leq C \prod_{i=1}^{m}\left\|f_{i}\right\|_{H_{\omega}^{p_{i}}} .
$$

(ii) If $\omega_{i} \in A_{1}$ for each $i$, then

$$
\|T(\vec{f})\|_{L_{\nu_{\vec{\omega}}}^{p}} \leq C \prod_{i=1}^{m}\left\|f_{i}\right\|_{H_{\omega_{i}}^{p_{i}}} .
$$

In order to state other results, let us first give some notations and definitions. Denote by $\vec{f}$ the $m$-tuple $\left(f_{1}, \ldots, f_{m}\right)$ and by $I_{\alpha}$ the $m$-linear fractional integral operator, and by $M(\vec{f})$ the $m$-sublinear maximal function, defined 
as follows:

$$
\begin{aligned}
& I_{\alpha}(\vec{f})(x)=\int f_{1}\left(x-\theta_{1} y\right) \cdots f_{m}\left(x-\theta_{m} y\right)|y|^{\alpha-n} d y, \\
& M(\vec{f})(x)=\sup _{N>0}\left(\Omega_{n} N^{n}\right)^{-1} \int\left|f_{1}\left(x-\theta_{1} y\right)\right| \cdots\left|f_{1}\left(x-\theta_{m} y\right)\right| d y,
\end{aligned}
$$

where $\theta_{k}, k=1, \ldots, m$, are fixed, distinct and nonzero real numbers. Note that if $m=1$, then the above operators coincide with the classical fractional integrals and Hardy-Littlewood maximal function.

In 1992, Grafakos 11 proved the $L^{p_{1}} \times \cdots \times L^{p_{k}}$ boundedness for the above class of multilinear fractional integrals $I_{\alpha}$. He also proved two multilinear versions of inequalities for multilinear fractional integrals related to the classical Trudinger inequality. We give a weighted version of an inequality in [1]:

TheOREM 1.2. Let $0<\alpha<n$ and $s=n / \alpha$ be the harmonic mean of $p_{1}, \ldots, p_{m}>1$. Let $B$ be a ball of radius $R$ in $\mathbb{R}^{n}$, let $f_{k} \in L_{\omega_{k}}^{p_{k}}\left(\mathbb{R}^{n}\right)$ be supported in $B$, and for any $x \in B$, let $\tilde{\nu}_{\vec{\omega}}=\prod_{k=1}^{m} \omega_{k}^{s / p_{k}}, \tilde{\nu}_{\vec{\omega}}(x) \geq 1$, and $\omega_{k} \in A_{s}$. Then for any $\gamma<1$, there exists a constant $C_{0}(\gamma)$ depending only on $n, \alpha$, the $\theta_{k}$ 's and $\gamma$, such that

$$
\begin{aligned}
\int_{B} \exp \left(\frac{n}{\omega_{n-1}} \gamma\left(\frac{L I_{\alpha}\left(f_{1}, \ldots, f_{m}\right)}{\left\|f_{1}\right\|_{L_{\omega_{1}}^{p_{1}}} \cdots\left\|f_{m}\right\|_{L_{\omega_{m}}^{p_{m}}}}\right)^{\frac{n}{n-\alpha}} \tilde{\nu}_{\vec{\omega}}\right) d x & \\
& \leq C_{0}(\gamma) \prod_{k=1}^{m} \omega_{k}(B)^{s / p_{k}}
\end{aligned}
$$

where $L=\prod_{k}\left|\theta_{k}\right|^{n / p_{k}}$.

REMARK. We note that if $\omega_{k}(x)=1$, then our result is the same as Theorem 3 in [11]. We give an example of $\tilde{\nu}_{\vec{\omega}}$ : Let $\omega_{k}(x)=(1+|x|)^{\alpha_{k}}$, $\alpha_{k} \geq 0$ (for each $k$ ); then $\tilde{\nu}_{\vec{\omega}}$ satisfies the conditions of Theorem 1.2.

Assume $m=1, \omega_{k}=1$. Trudinger [26] proved exponential integrability of $I_{\alpha}(\vec{f})$ for $\alpha=1$, and Strichartz [24] for other $\alpha$. In 1972, Hedberg [18] gave a simpler proof for all $\alpha$. In 1970, Hempel et al. [19] showed that if $\gamma>1$, then (1.8) cannot hold for $\alpha=1$, and later Adams [1] got the same conclusion for all $\alpha$; in the endpoint case $\gamma=1,(1.8)$ cannot hold either. In 1985, Chang and Marshall [2] proved a similar sharp exponential inequality concerning the Dirichlet integral. For $m \geq 2, w_{k}=1$, the result was obtained by Grafakos [1] as already mentioned above.

2. Proof of Theorem 1.1. We need the following lemma:

Lemma 2.1. Let $0<p \leq 1$. Then there is a constant $C=C(p)$ such that for all finite collections $\left\{Q_{k}\right\}_{k=1}^{m}$ of cubes in $\mathbb{R}^{n}$ and all nonnegative 
functions $g_{k} \in L_{\omega}$ with $\operatorname{supp} g_{k} \subset Q_{k}$ we have

$$
\left\|\sum_{k=1}^{m} g_{k}\right\|_{L_{\omega}^{p}} \leq C\left\|\sum_{k=1}^{m} \frac{1}{\omega\left(Q_{k}\right)} \int_{Q_{k}} g_{k}(x) \omega(x) d x \chi_{\tilde{Q}_{k}}\right\|_{L_{\omega}^{p}} .
$$

where $\tilde{Q}_{k}$ denotes the cube with the same center and $2 \sqrt{n}$ times the side length of $Q_{k}$, i.e. $l\left(\tilde{Q}_{k}\right)=2 \sqrt{n} l\left(Q_{k}\right)$.

The proof of this lemma can be easily obtained by replacing $L^{p}$ norm by $L_{\omega}^{p}$ norm and $\left(1 /\left|Q_{k}\right|\right) \int_{Q_{k}} g_{k}(x) d x$ by $\left(1 / \omega\left(Q_{k}\right)\right) \int_{Q_{k}} g_{k}(x) \omega d x$ in [13].

We will use the following facts:

(a) Let $q \geq 1$ and $\omega \in A_{q}$. Suppose $a$ is an $\omega$ - $(p, \infty, s)$-atom. Then by the definition, $a$ is supported in a cube $Q,\|a\|_{\infty} \leq \omega(Q)^{-1 / p}$, and $\int_{\mathbb{R}^{n}} a(y) y^{\alpha} d y=0$ for any multi-index $\alpha$ with $|\alpha| \leq s$. From $\|a\|_{\infty} \leq$ $\omega(Q)^{-1 / p}$ we get

$$
\|a\|_{L_{\omega}^{q}} \leq\left(\int_{Q}|a(y)|^{q} \omega(y) d y\right)^{1 / q} \leq\|a\|_{\infty} \omega(Q)^{1 / q} \leq \omega(Q)^{1 / q-1 / p},
$$

which means that $a$ is an $\omega$ - $(p, q, s)$-atom.

(b) From [10], we know that if $\omega \in A_{p}, p \geq 1$, then for any cube $Q$ and $\lambda>1$,

$$
\omega(\lambda Q) \leq C \lambda^{n p} \omega(Q),
$$

where $C$ does not depend on $Q$ nor on $\lambda$.

Now, we begin to prove Theorem 1.1. First, we consider case (i). Observe that if each $\omega_{i}$ is in $A_{p_{i}}$, then

$$
\prod_{i=1}^{m} A_{p_{i}} \subset A_{\vec{p}}
$$

so $\omega \times \cdots \times \omega \in A_{\vec{q}}$.

We use the atomic decomposition of $H_{\omega}^{p}$ spaces. Since $T$ is bounded from $L^{2 m} \times \cdots \times L^{2 m}$ into $L^{2}$, and since finite sums of atoms are dense in $H_{\omega}^{p}$, we will work with such sums and obtain estimates independent of the number of terms in each sum.

We can assume each $f_{i}, 1 \leq i \leq m$, is a finite sum of $H_{\omega}^{p_{i}}$-atoms, $f_{i}=$ $\sum_{k} \lambda_{i, k} a_{i, k}$, where $a_{i, k}$ are $\left(p_{i}, \infty, s\right)$-atoms, which means they are supported in cubes $Q_{i, k}$ and $\left|a_{i, k}\right| \leq \omega\left(Q_{i, k}\right)^{-1 / p_{i}}$. However, by the above fact (a),

$$
\begin{aligned}
& \left\|a_{i, k}\right\|_{L_{\omega}^{q_{i}}} \leq \omega\left(Q_{i, k}\right)^{1 / q_{i}-1 / p_{i}}, \\
& \int_{Q_{i, k}} a_{i, k}(x) x^{\alpha} d x=0, \quad|\alpha| \leq s, s \geq\left[n\left(\left(q_{i}\right)_{\omega} / p_{i}-1\right)\right] .
\end{aligned}
$$


Denote by $c_{i, k}$ and $l\left(Q_{i, k}\right)$ the center and the side length of $Q_{i, k}$, and let $\tilde{Q}_{i, k}=8 \sqrt{n} Q_{i, k}$. Employing multilinearity we write

$$
\begin{aligned}
T(\vec{f})(x) & =T\left(f_{1}, \ldots, f_{m}\right)(x) \\
& =\sum_{k_{1}} \cdots \sum_{k_{m}} \lambda_{1, k_{1}} \cdots \lambda_{m, k_{m}} T\left(a_{1, k_{1}}, \ldots, a_{m, k_{m}}\right)(x) .
\end{aligned}
$$

For $x \in \mathbb{R}^{n}$ and fixed $k_{1}, \ldots, k_{m}$, we estimate

$$
|T(\vec{f})(x)| \leq I_{1}(x)+I_{2}(x),
$$

where

$$
\begin{aligned}
& I_{1}(x)=\sum_{k_{1}} \cdots \sum_{k_{m}}\left|\lambda_{1, k_{1}}\right| \cdots\left|\lambda_{m, k_{m}}\right|\left|T\left(a_{1, k_{1}}, \ldots, a_{m, k_{m}}\right)(x)\right| \chi_{\tilde{Q}_{1, k_{1}} \cap \cdots \cap \tilde{Q}_{m, k_{m}}}, \\
& I_{2}(x)=\sum_{k_{1}} \cdots \sum_{k_{m}}\left|\lambda_{1, k_{1}}\right| \cdots\left|\lambda_{m, k_{m}}\right|\left|T\left(a_{1, k_{1}}, \ldots, a_{m, k_{m}}\right)(x)\right| \chi_{\tilde{Q}_{1, k_{1}}^{c} \cup \cdots \cup \tilde{Q}_{m, k_{m}}^{c}} .
\end{aligned}
$$

First, let us discuss $I_{1}(x)$. For fixed $k_{1}, \ldots, k_{m}$, assume that $\tilde{Q}_{1, k_{1}} \cap \cdots \cap$ $\tilde{Q}_{m, k_{m}} \neq \emptyset$, since otherwise there is nothing to prove.

Suppose that $Q_{i^{*}, k_{i^{*}}}, i^{*} \in\{1, \ldots, m\}$, has the smallest size among all these cubes. We take a cube $G_{k_{1}, \ldots, k_{m}}$ such that

$$
\tilde{Q}_{1, k_{1}} \cap \cdots \cap \tilde{Q}_{m, k_{m}} \subset G_{k_{1}, \ldots, k_{m}} \subset \tilde{G}_{k_{1}, \ldots, k_{m}} \subset \tilde{\tilde{Q}}_{1, k_{1}} \cap \cdots \cap \tilde{\tilde{Q}}_{m, k_{m}}
$$

and

$$
\omega\left(G_{k_{1}, \ldots, k_{m}}\right) \geq C \omega\left(Q_{i^{*}, k_{i^{*}}}\right) .
$$

Here $\tilde{G}_{k_{1}, \ldots, k_{m}}$ denotes the cube with the same center and $8 \sqrt{n}$ times the side length of the cube $G_{k_{1}, \ldots, k_{m}}$.

By Hölder's inequality, Theorem B, and (2.2), we have

$$
\begin{aligned}
& \frac{1}{\omega\left(G_{k_{1}, \ldots, k_{m}}\right)} \int_{G_{k_{1}, \ldots, k_{m}}}\left|T\left(a_{1, k_{1}}, \ldots, a_{m, k_{m}}\right)(x)\right| \omega(x) d x \\
\leq & \frac{1}{\omega\left(G_{k_{1}, \ldots, k_{m}}\right)} \omega\left(G_{k_{1}, \ldots, k_{m}}\right)^{1 / q^{\prime}}\left\|T\left(a_{1, k_{1}}, \ldots, a_{m, k_{m}}\right)(x)\right\|_{L_{\omega}^{q}} \\
\leq & C \omega\left(G_{k_{1}, \ldots, k_{m}}\right)^{-1 / q} \prod_{i=1}^{m}\left\|a_{i, k_{i}}\right\|_{L_{\omega}^{q_{i}}} \\
\leq & C \omega\left(G_{k_{1}, \ldots, k_{m}}\right)^{-1 / q} \prod_{i=1}^{m} \omega\left(Q_{i, k_{i}}\right)^{1 / q_{i}-1 / p_{i}} \\
\leq & C \prod_{i=1}^{m} \omega\left(G_{k_{1}, \ldots, k_{m}}\right)^{-1 / q_{i}} \prod_{i=1}^{m} \omega\left(Q_{i^{*}, k_{i^{*}}}\right)^{1 / q_{i}-1 / p_{i}}=C \prod_{i=1}^{m} \omega\left(Q_{i^{*}, k_{i^{*}}}\right)^{-1 / p_{i}} .
\end{aligned}
$$


Using Lemma 2.1, we have

$$
\begin{aligned}
\left\|I_{1}\right\|_{L_{\omega}^{p}} & \leq C\left\|\sum_{k_{1}} \cdots \sum_{k_{m}}\left|\lambda_{1, k_{1}}\right| \cdots\left|\lambda_{m, k_{m}}\right| \prod_{i=1}^{m} \omega\left(Q_{i^{*}, k_{i^{*}}}\right)^{-1 / p_{i}} \chi_{\tilde{G}_{k_{1}, \ldots, k_{m}}}\right\|_{L_{\omega}^{p}} \\
& \leq C\left\|\prod_{i=1}^{m}\left(\sum_{k_{i}}\left|\lambda_{i, k_{i}}\right| \omega\left(Q_{i^{*}, k_{i^{*}}}\right)^{-1 / p_{i}} \chi_{\tilde{G}_{k_{1}, \ldots, k_{m}}}\right)\right\|_{L_{\omega}^{p}} \\
& \leq C\left\|\prod_{i=1}^{m}\left(\sum_{k_{i}}\left|\lambda_{i, k_{i}}\right| \omega\left(Q_{i^{*}, k_{i^{*}}}\right)^{-1 / p_{i}} \omega(x)^{1 / p_{i}} \chi_{\tilde{G}_{k_{1}, \ldots, k_{m}}}\right)\right\|_{L^{p}} \\
& \leq C \prod_{i=1}^{m}\left\|\left(\sum_{k_{i}}\left|\lambda_{i, k_{i}}\right| \omega\left(Q_{i^{*}, k_{i^{*}}}\right)^{-1 / p_{i}} \omega(x)^{1 / p_{i}} \chi_{\tilde{G}_{k_{1}, \ldots, k_{m}}}\right)\right\|_{L^{p_{i}}} \\
& \leq C \prod_{i=1}^{m}\left(\sum_{k_{i}}\left|\lambda_{i, k_{i}}\right|^{p_{i}} \omega\left(Q_{i^{*}, k_{i}}\right)^{-1} \omega\left(\tilde{\tilde{Q}}_{i^{*}, k_{i^{*}}}\right)\right)^{1 / p_{i}} \\
& =C \prod_{i=1}^{m}\left(\sum_{k_{i}}\left|\lambda_{i, k_{i}}\right|^{p_{i}}\right)^{1 / p_{i}} .
\end{aligned}
$$

Secondly, we estimate $I_{2}(x)$. Let $A$ be a nonempty subset of $\{1, \ldots, m\}$, of cardinality $|A|$, so $1 \leq|A| \leq m$. Let $A^{c}=\{1, \ldots, m\} \backslash A$. If $A=$ $\{1, \ldots, m\}$, we define

$$
\left(\bigcap_{i \in A} \tilde{Q}_{i, k_{i}}^{c}\right) \cap\left(\bigcap_{i \in A^{c}} \tilde{Q}_{i, k_{i}}\right)=\bigcap_{i \in A} \tilde{Q}_{i, k_{i}}^{c}
$$

Then

$$
\tilde{Q}_{1, k_{1}}^{c} \cup \cdots \cup \tilde{Q}_{m, k_{m}}^{c}=\bigcup_{A \subset\{1, \ldots, m\}}\left(\left(\bigcap_{i \in A} \tilde{Q}_{i, k_{i}}^{c}\right) \cap\left(\bigcap_{i \in A^{c}} \tilde{Q}_{i, k_{i}}\right)\right) .
$$

We set

$$
E_{A}=\left(\bigcap_{i \in A} \tilde{Q}_{i, k_{i}}^{c}\right) \cap\left(\bigcap_{i \in A^{c}} \tilde{Q}_{i, k_{i}}\right) .
$$

For fixed $A$, we assume that the side length of the cube $Q_{i^{*}, k_{i}}, i^{*} \in$ $A$, is the smallest among the side lengths of the cubes $Q_{i, k_{i}}, i \in A$. Let $P_{c_{i^{*}, k_{i^{*}}}^{N}}\left(x, y_{1}, \ldots, y_{m}\right)$ be the $N$ th order Taylor polynomial of $K\left(x, y_{1}, \ldots, y_{m}\right)$ with respect to the variable $y_{i^{*}}$ at the point $c_{i^{*}, k_{i^{*}}}$.

Since $a_{i^{*}, k_{i^{*}}}$ has zero vanishing moments up to $N$, by (1.3) we get

$$
\begin{aligned}
\left|T\left(a_{1, k_{1}}, \ldots, a_{m, k_{m}}\right)(x)\right|= & \mid \int_{\left(\mathbb{R}^{n}\right)^{m-1}} \prod_{i=1, i \neq i^{*}}^{m} a_{i, k_{i}}\left(y_{i}\right) \int_{\mathbb{R}^{n}} a_{i^{*}, k_{i^{*}}}\left(y_{i^{*}}\right) \\
& \times\left(K\left(x, y_{1}, \ldots, y_{m}\right)-P_{c_{i^{*}, k_{i^{*}}}^{N}}^{N}\left(x, y_{1}, \ldots, y_{m}\right)\right) d \vec{y} \mid
\end{aligned}
$$




$$
\begin{aligned}
\leq & C \int_{\left(\mathbb{R}^{n}\right)^{m-1}} \prod_{i=1, i \neq i^{*}}^{m}\left|a_{i, k_{i}}\left(y_{i}\right)\right| \int_{\mathbb{R}^{n}}\left|a_{i^{*}, k_{i^{*}}}\left(y_{i^{*}}\right)\right|\left|y_{i^{*}}-c_{i^{*}, k_{i}}\right|^{N+1} \\
& \times\left(|x-\xi|+\sum_{j=1, j \neq i^{*}}^{m}\left|x-y_{j}\right|\right)^{-m n-N-1} d \vec{y}
\end{aligned}
$$

where $\xi$ is between $y_{i^{*}}$ and $c_{i^{*}, k_{i^{*}}}$.

For $x \in\left(\bigcap_{i \in A} \tilde{Q}_{i, k_{i}}^{c}\right) \cap\left(\bigcap_{i \in A^{c}} \tilde{Q}_{i, k_{i}}\right)$,

$$
|x-\xi| \geq\left|x-c_{i^{*}, k_{i^{*}}}\right|-\left|\xi-c_{i^{*}, k_{i^{*}}}\right| \geq \frac{1}{2}\left|x-c_{i^{*}, k_{i^{*}}}\right|
$$

since $x \notin \tilde{Q}_{i^{*}, k_{i^{*}}}$.

Similarly, $\left|x-y_{i}\right| \geq \frac{1}{2}\left|x-c_{i, k_{i}}\right|$ for $y_{i} \in Q_{i, k_{i}}, i \in A \backslash\left\{i^{*}\right\}$.

Note the following two facts:

(c) If $a$ is an $\omega$ - $(p, \infty, s)$-atom supported on a cube $Q$, and $M>n$, then for any positive number $b$ and $c \in \mathbb{R}^{n}$,

$$
\int_{\mathbb{R}^{n}} \frac{|a(y)|}{(b+|y-c|)^{M}} d y \leq b^{n} \omega(Q)^{-1 / p} \int_{\mathbb{R}^{n}} \frac{1}{(1+|y|)^{M}} d y .
$$

(d) By the definition, for $\omega \in A_{p}$ we have

$$
\left(\int_{Q} \omega(y)^{1-p^{\prime}} d y\right)^{p-1} \leq C|Q|^{p}\left(\int_{Q} \omega(y) d y\right)^{-1} .
$$

Using Hölder's inequality, (2.2) and (c)-(d) we obtain

$$
\begin{aligned}
& \left|T\left(a_{1, k_{1}}, \ldots, a_{m, k_{m}}\right)(x)\right| \\
& \leq C \prod_{i \in A}\left(\left\|a_{i, k_{i}}\right\|_{L_{\omega}^{q_{i}}}\left(\int_{Q_{i, k_{i}}} \omega^{-q_{i}^{\prime} / q_{i}}\right)^{1 / q_{i}^{\prime}}\right)\left|Q_{i^{*}, k_{i^{*}}}\right|^{(N+1) / n} \\
& \times\left(\frac{1}{2} \sum_{i \in A}\left|x-c_{i, k_{i}}\right|\right)^{-m n-N-1+n(m-|A|)} \prod_{i \in A^{c}} \omega\left(Q_{i, k_{i}}\right)^{-1 / p_{i}} \\
& \leq C \prod_{i \in A}\left(\omega\left(Q_{i, k_{i}}\right)^{1 / q_{i}-1 / p_{i}}\left(\int_{Q_{i, k_{i}}} \omega^{1-q_{i}^{\prime}}\right)^{\left(q_{i}-1\right) / q_{i}}\right)\left|Q_{i^{*}, k_{i^{*}}}\right|^{(N+1) / n} \\
& \times\left(\sum_{i \in A}\left|x-c_{i, k_{i}}\right|\right)^{-m n-N-1+n(m-|A|)} \prod_{i \in A^{c}} \omega\left(Q_{i, k_{i}}\right)^{-1 / p_{i}} \\
& \leq C \prod_{i \in A}\left(\omega\left(Q_{i, k_{i}}\right)^{1 / q_{i}-1 / p_{i}}\left|Q_{i, k_{i}}\right| \omega\left(Q_{i, k_{i}}\right)^{-1 / q_{i}}\right)\left|Q_{i^{*}, k_{i^{*}}}\right|^{(N+1) / n} \\
& \times\left(\sum_{i \in A}\left|x-c_{i, k_{i}}\right|\right)^{-m n-N-1+n(m-|A|)} \prod_{i \in A^{c}} \omega\left(Q_{i, k_{i}}\right)^{-1 / p_{i}}
\end{aligned}
$$




$$
\begin{aligned}
\leq & C \prod_{i \in A}\left(\omega\left(Q_{i, k_{i}}\right)^{-1 / p_{i}}\left|Q_{i, k_{i}}\right|\right)\left|Q_{i^{*}, k_{i^{*}}}\right|^{(N+1) / n} \\
& \times\left(\sum_{i \in A}\left|x-c_{i, k_{i}}\right|\right)^{-m n-N-1+n(m-|A|)} \prod_{i \in A^{c}} \omega\left(Q_{i, k_{i}}\right)^{-1 / p_{i}} .
\end{aligned}
$$

Since $Q_{i^{*}, k_{i^{*}}}$ is the smallest cube among $\left\{Q_{i, k_{i}}\right\}_{i \in A}$, and $x \in \bigcap_{i \in A^{c}} Q_{i, k_{i}}$, we get

$$
\begin{aligned}
\mid T\left(a_{1, k_{1}}, \ldots,\right. & \left.a_{m, k_{m}}\right)(x) \mid \\
& \leq C \prod_{i \in A} \frac{\omega\left(Q_{i, k_{i}}\right)^{-1 / p_{i}}\left|Q_{i, k_{i}}\right|\left|Q_{i, k_{i}}\right|^{\frac{N+1}{n|A|}}}{\left(\left|x-c_{i, k_{i}}\right|+l\left(Q_{i, k_{i}}\right)\right)^{n+\frac{N+1}{|A|}}} \prod_{i \in A^{c}} \omega\left(Q_{i, k_{i}}\right)^{-1 / p_{i}} \\
& \leq C \prod_{i=1}^{m} \frac{\omega\left(Q_{i, k_{i}}\right)^{-1 / p_{i}}\left|Q_{i, k_{i}}\right|\left|Q_{i, k_{i}}\right|^{\frac{N+1}{n|A|}}}{\left(\left|x-c_{i, k_{i}}\right|+l\left(Q_{i, k_{i}}\right)\right)^{n+\frac{N+1}{|A|}}}
\end{aligned}
$$

Hence, by Minkowski's inequality and Hölder's inequality, we deduce that

$$
\begin{aligned}
& \left\|I_{2}\right\|_{L_{\omega}^{p}} \leq C \| \sum_{k_{1}} \cdots \sum_{k_{m}}\left|\lambda_{1, k_{1}}\right| \cdots\left|\lambda_{m, k_{m}}\right| \\
& \times \sum_{A \subset\{1, \ldots, m\}} \prod_{i=1}^{m} \frac{\omega\left(Q_{i, k_{i}}\right)^{-1 / p_{i}}\left|Q_{i, k_{i}}\right|\left|Q_{i, k_{i}}\right|^{\frac{N+1}{n|A|}}}{\left(\left|x-c_{i, k_{i}}\right|+l\left(Q_{i, k_{i}}\right)\right)^{n+\frac{N+1}{|A|}}} \chi_{E_{A}} \|_{L_{\omega}^{p}} \\
& \leq C \sum_{A \subset\{1, \ldots, m\}}\left\|\prod_{i=1}^{m}\left(\sum_{k_{i}}\left|\lambda_{i, k_{i}}\right| \frac{\omega\left(Q_{i, k_{i}}\right)^{-1 / p_{i}}\left|Q_{i, k_{i}}\right|\left|Q_{i, k_{i}}\right|^{\frac{N+1}{n|A|}}}{\left(\left|x-c_{i, k_{i}}\right|+l\left(Q_{i, k_{i}}\right)\right)^{n+\frac{N+1}{|A|}}}\right) \chi_{E_{A}}\right\|_{L_{\omega}^{p}} \\
& \leq C \sum_{A \subset\{1, \ldots, m\}} \prod_{i=1}^{m}\left\|\sum_{k_{i}}\left|\lambda_{i, k_{i}}\right| \frac{\omega\left(Q_{i, k_{i}}\right)^{-1 / p_{i}}\left|Q_{i, k_{i}}\right|\left|Q_{i, k_{i}}\right|^{\frac{N+1}{n|A|}}}{\left(\left|x-c_{i, k_{i}}\right|+l\left(Q_{i, k_{i}}\right)\right)^{n+\frac{N+1}{|A|}}} \omega(x)^{1 / p_{i}} \chi_{E_{A}}\right\|_{L^{p_{i}}} \\
& \leq C \prod_{i=1}^{m}\left(\sum_{k_{i}}\left|\lambda_{i, k_{i}}\right|^{p_{i}}\left\|\frac{\omega\left(Q_{i, k_{i}}\right)^{-1 / p_{i}}\left|Q_{i, k_{i}}\right|\left|Q_{i, k_{i}}\right|^{\frac{N+1}{n|A|}}}{\left(\left|x-c_{i, k_{i}}\right|+l\left(Q_{i, k_{i}}\right)\right)^{n+\frac{N+1}{|A|}}} \omega(x)^{1 / p_{i}} \chi_{E_{A}}\right\|_{L^{p_{i}}}\right)^{p_{i}}
\end{aligned}
$$

where we used the $p_{i}$-subadditivity of the $L^{p_{i}}$ quasi-norm. So we only have to estimate

$$
J_{i}:=\int_{\mathbb{R}^{n}} \frac{\omega\left(Q_{i, k_{i}}\right)^{-1}\left|Q_{i, k_{i}}\right|^{p_{i}}\left|Q_{i, k_{i}}\right|^{\frac{N+1}{n|A|} p_{i}}}{\left(\left|x-c_{i, k_{i}}\right|+l\left(Q_{i, k_{i}}\right)\right)^{n p_{i}+\frac{N+1}{|A|} p_{i}}} \chi_{E_{A}}(x) \omega(x) d x .
$$

If $i \in A$, then

$$
J_{i} \leq C \sum_{j=1}^{\infty} \int_{2^{j} \tilde{Q}_{i, k_{i}} \backslash 2^{j-1} \tilde{Q}_{i, k_{i}}} \frac{\omega\left(Q_{i, k_{i}}\right)^{-1}\left|Q_{i, k_{i}}\right|^{p_{i}}\left|Q_{i, k_{i}}\right|^{\frac{N+1}{n|A|} p_{i}}}{\left(\left|x-c_{i, k_{i}}\right|+l\left(Q_{i, k_{i}}\right)\right)^{n p_{i}+\frac{N+1}{|A|} p_{i}}} \omega(x) d x
$$




$$
\begin{aligned}
& \leq C \omega\left(Q_{i, k_{i}}\right)^{-1}\left|Q_{i, k_{i}}\right|^{p_{i}}\left|Q_{i, k_{i}}\right|^{\frac{N+1}{n|A|} p_{i}} \sum_{j=1}^{\infty} \frac{\omega\left(2^{j} \tilde{Q}_{i, k_{i}}\right)}{\left(2^{j-1}\left|\tilde{Q}_{i, k_{i}}\right|^{1 / n}\right)^{n p_{i}+\frac{N+1}{|A|} p_{i}}} \\
& \leq C \omega\left(Q_{i, k_{i}}\right)^{-1}\left|Q_{i, k_{i}}\right|^{p_{i}}\left|Q_{i, k_{i}}\right|^{\frac{N+1}{n|A|} p_{i}} \sum_{j=1}^{\infty} \frac{2^{j n q_{i}} \omega\left(\tilde{Q}_{i, k_{i}}\right)}{2^{(j-1)\left(n p_{i}+\frac{N+1}{|A|} p_{i}\right)}\left|\tilde{Q}_{i, k_{i}}\right|^{p_{i}+\frac{N+1}{n|A|} p_{i}}} \\
& \leq C .
\end{aligned}
$$

To show the last inequality, we used $N>m n\left(q_{i} / p_{i}-1\right)-1$. If $i \in A^{c}$, then

$$
\begin{aligned}
& J_{i} \leq C \int_{\tilde{Q}_{i, k_{i}}} \frac{\omega\left(Q_{i, k_{i}}\right)^{-1}\left|Q_{i, k_{i}}\right|^{p_{i}}\left|Q_{i, k_{i}}\right|^{\frac{N+1}{n|A|} p_{i}}}{\left(\left|x-c_{i, k_{i}}\right|+l\left(Q_{i, k_{i}}\right)\right)^{n p_{i}+\frac{N+1}{|A|} p_{i}}} \omega(x) d x \\
& \leq C \omega\left(Q_{i, k_{i}}\right)^{-1}\left|Q_{i, k_{i}}\right|^{p_{i}}\left|Q_{i, k_{i}}\right|^{\frac{N+1}{n|A|} p_{i}} \frac{\omega\left(\tilde{Q}_{i, k_{i}}\right)}{\left(\left|Q_{i, k_{i}}\right|^{1 / n}\right)^{n p_{i}+\frac{N+1}{|A|} p_{i}}} \leq C .
\end{aligned}
$$

Thus we have

$$
\left\|I_{2}\right\|_{L_{\omega}^{p}} \leq C \prod_{i=1}^{m}\left(\sum_{k_{i}}\left|\lambda_{i, k_{i}}\right|^{p_{i}}\right)^{1 / p_{i}}
$$

In conclusion, summing the estimates for $I_{1}$ and $I_{2}$, and taking the limit, we obtain

$$
\|T(\vec{f})\|_{L_{\nu_{\vec{\omega}}}^{p}} \leq C \prod_{i=1}^{m}\left\|f_{i}\right\|_{H_{\omega_{i}}^{p_{i}}}
$$

This completes the proof of Theorem 1.1(i).

For (ii), the procedure is similar; we only show the differences.

For $I_{1}(x)$, we take a cube $G_{k_{1}, \ldots, k_{m}}$ such that $\omega_{i}\left(G_{k_{1}, \ldots, k_{m}}\right) \geq C \omega_{i}\left(Q_{i^{*}, k_{i}}\right)$ for each $i$. Thus

$$
\begin{aligned}
& \frac{1}{\nu_{\vec{\omega}}\left(G_{k_{1}, \ldots, k_{m}}\right)} \int_{G_{k_{1}, \ldots, k_{m}}}\left|T\left(a_{1, k_{1}}, \ldots, a_{m, k_{m}}\right)(x)\right| \nu_{\vec{\omega}}(x) d x \\
& \leq C \nu_{\vec{\omega}}\left(G_{k_{1}, \ldots, k_{m}}\right)^{-1 / q} \prod_{i=1}^{m}\left\|a_{i, k_{i}}\right\|_{L_{\omega_{i}}^{q_{i}}} \\
& \leq C\left(\prod_{i=1}^{m}\left|G_{k_{1}, \ldots, k_{m}}\right|^{1 / q_{i}} \underset{x \in G_{k_{1}, \ldots, k_{m}}}{\operatorname{ess}} \inf _{i}(x)^{1 / q_{i}}\right)^{-1} \prod_{i=1}^{m} \omega\left(Q_{i^{*}, k_{i}}\right)^{1 / q_{i}-1 / p_{i}} \\
& \leq C\left(\prod_{i=1}^{m} \omega_{i}\left(G_{k_{1}, \ldots, k_{m}}\right)^{1 / q_{i}}\right)^{-1} \prod_{i=1}^{m} \omega\left(Q_{i^{*}, k_{i} *}\right)^{1 / q_{i}-1 / p_{i}} \leq C \prod_{i=1}^{m} \omega\left(Q_{i^{*}, k_{i^{*}}}\right)^{-1 / p_{i}}
\end{aligned}
$$

Hence, by Lemma 2.1,

$$
\left\|I_{1}\right\|_{L_{\nu_{\vec{\omega}}}^{p}} \leq C\left\|\sum_{k_{1}} \cdots \sum_{k_{m}}\left|\lambda_{1, k_{1}}\right| \cdots\left|\lambda_{m, k_{m}}\right| \prod_{i=1}^{m} \omega_{i}\left(Q_{i^{*}, k_{i^{*}}}\right)^{-1 / p_{i}} \chi_{\tilde{G}_{k_{1}, \ldots, k_{m}}}\right\|_{L_{\nu_{\vec{\omega}}}^{p}}
$$




$$
\begin{aligned}
& \leq C \prod_{i=1}^{m}\left\|\left(\sum_{k_{i}}\left|\lambda_{i, k_{i}}\right| \omega_{i}\left(Q_{i^{*}, k_{i^{*}}}\right)^{-1 / p_{i}} \omega_{i}(x)^{1 / p_{i}} \chi_{\tilde{G}_{k_{1}, \ldots, k_{m}}}\right)\right\|_{L^{p_{i}}} \\
& \leq C \prod_{i=1}^{m}\left(\sum_{k_{i}}\left|\lambda_{i, k_{i}}\right|^{p_{i}}\right)^{1 / p_{i}} .
\end{aligned}
$$

For $I_{2}(x)$,

$$
\left|T\left(a_{1, k_{1}}, \ldots, a_{m, k_{m}}\right)(x)\right| \leq C \prod_{i=1}^{m} \frac{\omega_{i}\left(Q_{i, k_{i}}\right)^{-1 / p_{i}}\left|Q_{i, k_{i}}\right|\left|Q_{i, k_{i}}\right|^{\frac{N+1}{n|A|}}}{\left(\left|x-c_{i, k_{i}}\right|+l\left(Q_{i, k_{i}}\right)\right)^{n+\frac{N+1}{|A|}}}
$$

and so

$$
\begin{aligned}
\left\|I_{2}\right\|_{L_{\nu_{\vec{\omega}}}^{p}} \leq & C \| \sum_{k_{1}} \cdots \sum_{k_{m}}\left|\lambda_{1, k_{1}}\right| \cdots\left|\lambda_{m, k_{m}}\right| \\
& \times \sum_{A \subset\{1, \ldots, m\}} \prod_{i=1}^{m} \frac{\omega_{i}\left(Q_{i, k_{i}}\right)^{-1 / p_{i}}\left|Q_{i, k_{i}}\right|\left|Q_{i, k_{i}}\right|^{\frac{N+1}{n|A|}}}{\left(\left|x-c_{i, k_{i}}\right|+l\left(Q_{i, k_{i}}\right)\right)^{n+\frac{N+1}{|A|}}} \chi_{E_{A}} \|_{L_{\omega_{i}}^{p}} \\
\leq & C \prod_{i=1}^{m}\left(\sum_{k_{i}}\left|\lambda_{i, k_{i}}\right|^{p_{i}}\right)^{1 / p_{i}} .
\end{aligned}
$$

This completes the proof of case (ii).

REMARK. It is worth noting that our assumptions in Theorem 1.1 are in a sense natural ones. In fact, if we want to show the estimate

$$
\|T(\vec{f})\|_{L_{\nu_{\vec{\omega}}}^{p}} \leq C \prod_{i=1}^{m}\left\|f_{i}\right\|_{H_{\omega_{i}}^{p_{i}}}
$$

using the same procedure as in the proof of Theorem 1.1, then we have to replace $\omega$ with $\nu_{\vec{\omega}}$ in $(*)$, and get

$$
\begin{aligned}
\frac{1}{\nu_{\vec{\omega}}\left(G_{k_{1}, \ldots, k_{m}}\right)} \int_{G_{k_{1}, \ldots, k_{m}}} \mid T\left(a_{1, k_{1}}, \ldots,\right. & \left.a_{m, k_{m}}\right)(x) \mid \nu_{\vec{\omega}}(x) d x \\
& \leq C \nu_{\vec{\omega}}\left(G_{k_{1}, \ldots, k_{m}}\right)^{-1 / q} \prod_{i=1}^{m}\left\|a_{i, k_{i}}\right\|_{L_{\omega_{i}}}^{q_{i}} .
\end{aligned}
$$

So, we must require

$$
\nu_{\vec{\omega}}\left(G_{k_{1}, \ldots, k_{m}}\right)^{-1 / q} \leq C_{0} \prod_{i=1}^{m} \omega_{i}\left(G_{k_{1}, \ldots, k_{m}}\right)^{-1 / q_{i}} .
$$

However, by using Hölder's inequality, we know

$$
\prod_{i=1}^{m} \omega_{i}\left(G_{k_{1}, \ldots, k_{m}}\right)^{-1 / q_{i}} \leq \nu_{\vec{\omega}}\left(G_{k_{1}, \ldots, k_{m}}\right)^{-1 / q} \text {. }
$$


So, if $C_{0}=1$, we get

$$
\prod_{i=1}^{m} \omega_{i}\left(G_{k_{1}, \ldots, k_{m}}\right)^{-1 / q_{i}}=\nu_{\vec{\omega}}\left(G_{k_{1}, \ldots, k_{m}}\right)^{-1 / q},
$$

and hence we obtain $c_{1} \omega_{1}=\cdots=c_{m} \omega_{m}$ (see [12, p. 10]). For convenience, we assumed $c_{1}=\cdots=c_{m}=1$ in case (i) of Theorem 1.1. Another possibility for the case $C_{0}>1$ is the assumption in Theorem 1.1(ii).

3. Proof of Theorem 1.2. We follow the proof of Theorem 3 in [11]. We only need to prove Theorem 1.2 for $R=R_{0}$ with a constant $C_{0}^{\prime}(\gamma)$, since if we can do this, we obtain it for other values of $R$ with constant $C_{0}^{\prime}(\gamma)\left(R / R_{0}\right)^{n}$.

Let $R_{0}=1 / P$, where $P=2 \min \left|\theta_{k}\right|^{-1}$. We assume that the radius of $B$ is $R_{0}$. Without loss of generality, we assume that $f_{k} \geq 0$ and $\left\|f_{k}\right\|_{L_{w_{k}}^{p_{k}}}=1$.

Now, fix $x \in B$. The following estimate is known from [11, p. 53]:

$$
I_{\alpha}(\vec{f})(x) \leq C \delta^{\alpha} M(\vec{f})(x)+\int_{|y| \geq \delta} \prod f_{k}\left(x-\theta_{k} y\right)|y|^{\alpha-n} d y .
$$

Since all $f_{k}$ are supported in the ball $B$ and $x \in B$, the integral in (3.1) is over the set $\left\{y: \delta \leq|y| \leq P R_{0}=1\right\}$. For any $x \in B$ with $\tilde{\nu}_{\vec{\omega}}(x) \geq 1$, we deduce by Hölder's inequality that

$$
\begin{aligned}
& \int_{\delta \leq|y| \leq 1} \prod_{k=1}^{m} f_{k}\left(x-\theta_{k} y\right)|y|^{\alpha-n} d y \\
\leq & \left(\int_{\delta \leq|y| \leq 1} \prod\left(f_{k}\left(x-\theta_{k} y\right)\right)^{s} d y\right)^{1 / s}\left(\int_{\delta \leq|y| \leq 1}\left(|y|^{\alpha-n}\right)^{s^{\prime}} d y\right)^{1 / s^{\prime}} \\
\leq & \left(\int_{\delta \leq|y| \leq 1} \prod_{k=1}^{m}\left(f_{k}\left(x-\theta_{k} y\right)\right)^{s} \tilde{\nu}_{\vec{\omega}}\left(x-\theta_{k} y\right) d y\right)^{1 / s}\left(\int_{\delta \leq|y| \leq 1}\left(|y|^{\alpha-n}\right)^{\frac{n}{n-\alpha}} d y\right)^{\frac{n-\alpha}{n}} \\
\leq & \prod_{k=1}^{m}\left(\int_{\delta \leq|y| \leq 1}\left(f_{k}\left(x-\theta_{k} y\right)\right)^{p_{k}} \omega_{k}\left(x-\theta_{k} y\right) d y\right)^{1 / p_{k}}\left(\int_{\delta \leq|y| \leq 1}|y|^{-n} d y\right)^{\frac{n-\alpha}{n}} \\
\leq & L^{-1} \prod_{k=1}^{m}\left\|f_{k}\right\|_{L_{\omega_{k}}^{p_{k}}}\left(\int_{\delta \leq|y| \leq 1}|y|^{-n} d y\right)^{\frac{n-\alpha}{n}}=L^{-1}\left(\omega_{n-1} \ln \frac{1}{\delta}\right)^{\frac{n-\alpha}{n}} .
\end{aligned}
$$

Thus we have

$$
I_{\alpha}(\vec{f})(x) \leq C \delta^{\alpha} M(\vec{f})(x)+L^{-1}\left(\omega_{n-1} \ln \frac{1}{\delta}\right)^{\frac{n-\alpha}{n}} .
$$


Picking $\delta=1$, we get $I_{\alpha}(\vec{f})(x) \leq C M(\vec{f})(x)$ for all $x \in B$. Hence

$$
\delta=\epsilon\left(I_{\alpha}(\vec{f})(x)(C M(\vec{f})(x))^{-1}\right)^{1 / \alpha}
$$

will satisfy $\delta \leq 1$ for all $\epsilon \leq 1$. Thus using (3.2), we obtain

$$
I_{\alpha}(\vec{f})(x) \leq C \delta^{\alpha} M(\vec{f})(x)+L^{-1}\left(\frac{\omega_{n-1}}{n} \ln \left(\frac{(C M(f)(x))^{n / \alpha}}{\epsilon^{n} I_{\alpha}(f)(x)^{n / \alpha}}\right)\right)^{\frac{n-\alpha}{n}} .
$$

Simple computation gives

$$
\frac{\omega_{n-1}}{n} \gamma\left(L I_{\alpha}(\vec{f})(x)\right)^{n /(n-\alpha)} \leq \ln \frac{(C M(f)(x))^{n / \alpha}}{\epsilon^{n} I_{\alpha}(\vec{f})(x)^{n / \alpha}},
$$

where $\gamma=\left(1-\epsilon^{\alpha}\right)^{n /(n-\alpha)}$. We multiply both sides of (3.3) by $\tilde{\nu}_{\vec{\omega}}$, exponentiate and integrate over the set $B_{1}=\left\{x \in B: I_{\alpha}(f)(x) \geq 1\right\}$. Using $\omega_{k} \in A_{s}$ and denoting the Hardy-Littlewood maximal function by $\bar{M}$, we obtain

$$
\begin{aligned}
& \int_{B_{1}} \exp \left(\frac{\omega_{n-1}}{n} \gamma\left(L I_{\alpha}(\vec{f})(x)\right)^{n /(n-\alpha)} \tilde{\nu}_{\vec{\omega}}(x)\right) d x \\
& \leq \frac{1}{\epsilon^{n}} \int_{B_{1}} \frac{(C M(\vec{f})(x))^{n / \alpha} \prod_{k=1}^{m} \omega_{k}^{s / p_{k}}}{I_{\alpha}(\vec{f})(x)^{n / \alpha}} d x \leq \frac{C_{1}}{\epsilon^{n}} \int_{B_{1}}(M(\vec{f})(x))^{s} \prod \omega_{k}(x)^{s / p_{k}} d x \\
& \leq \frac{C_{1}}{\epsilon^{n}} \int_{B_{1}} \prod_{k=1}^{m}\left(\bar{M}\left(f_{k}^{p_{k} / s}\right)(x)\right)^{s^{2} / p_{k}} \prod \omega_{k}(x)^{s / p_{k}} d x \\
& \leq \frac{C_{1}}{\epsilon^{n}} \prod_{k=1}^{m}\left(\int_{B_{1}}\left(\bar{M}\left(f_{k}^{p_{k} / s}\right)(x)\right)^{s} \omega_{k}(x) d x\right)^{s / p_{k}} \\
& \leq \frac{C_{2}}{\epsilon^{n}} \prod_{k=1}^{m}\left(\left\|f_{k}^{p_{k} / s}\right\|_{L_{\omega_{k}}^{s}\left(B_{1}\right)}\right)^{s^{2} / p_{k}}=\frac{C_{2}}{\epsilon^{n}} \prod_{k=1}^{m}\left(\int_{B_{1}}\left(f_{k}^{p_{k} / s}(x)\right)^{s} \omega_{k}(x) d x\right)^{s / p_{k}} \\
& =\frac{C_{2}}{\epsilon^{n}} \prod_{k=1}^{m}\left(\int_{B_{1}} f_{k}^{p_{k}}(x) \omega_{k}(x) d x\right)^{s / p_{k}}=\frac{C_{2}}{\epsilon^{n}} \prod_{k=1}^{m}\left\|f_{k}\right\|_{L_{\omega_{k}}^{p_{k}}}^{s}
\end{aligned}
$$

Using the same method over the set $B_{2}=B \backslash B_{1}$, we easily obtain

$$
\begin{aligned}
\int_{B_{2}} \exp \left(\frac{\omega_{n-1}}{n}\right. & \left.\gamma\left(L I_{\alpha}(\vec{f})(x)\right)^{n /(n-\alpha)} \tilde{\nu}_{\vec{\omega}}(x)\right) d x \\
\leq & e^{n / \omega_{n-1} L^{n /(n-\alpha)}} \tilde{\nu}_{\vec{\omega}}\left(B_{2}\right) \leq C_{3} \prod_{k=1}^{m} \omega_{k}\left(B_{2}\right)^{s / p_{k}}=C_{4} .
\end{aligned}
$$

Combining the integrals over $B_{1}$ and $B_{2}$, we get the required inequality (1.8) with a constant $C_{0}^{\prime}(\gamma)=\max \left(C_{2}, C_{4}\right)\left(1+\left(1-\gamma^{(n-\alpha) / n}\right)\right)^{-n / \alpha}$, and $C_{0}(\gamma)=C_{0}^{\prime} \prod_{k=1}^{m} \omega_{k}\left(B_{2}\right)^{-s / p_{k}}$. 
Acknowledgements. The authors want to express their sincere thanks to the referee for his or her valuable remarks and suggestions. The authors also wish to thank Jerzy Trzeciak for his kind remarks which made this paper more readable.

The second named author was supported partly by NSFC (Grant No. 10701010), NSFC (Key program Grant No. 10931001), CPDRFSFP (Grant No. 200902070), Beijing Natural Science Foundation (Grant: 1102023).

The third named author was supported partly by Grant-in-Aid for Scientific Research (C) No. 20540195, Japan Society for the Promotion of Science.

\section{References}

[1] D. Adams, A sharp inequality of J. Moser for higher order derivatives, Ann. of Math. 128 (1988), 385-398.

[2] S.-Y. A. Chang and D. E. Marshall, On a sharp inequality concerning the Dirichlet integral, Amer. J. Math. 107 (1985), 1015-1033.

[3] X. Chen and Q. Xue, Weighted estimates for a class of multilinear fractional type operators, J. Math. Anal. Appl. 362 (2010), 355-373.

[4] M. Christ and J.-L. Journé, Polynomial growth estimates for multilinear singular integral operators, Acta Math. 159 (1987), 51-80.

[5] R. R. Coifman and Y. Meyer, On commutators of singular integrals and bilinear singular integrals, Trans. Amer. Math. Soc. 212 (1975), 315-331.

[6] - - - Commutateurs d'intégrales singulières et opérateurs multilinéaires, Ann. Inst. Fourier (Grenoble) 28 (1978), no. 3, 177-202.

[7] Y. Ding, M. Y. Lee and C. C. Lin, Marcinkiewicz integral on weighted Hardy spaces, Arch. Math. (Basel) 80 (2003), 620-629.

[8] J. Duoandikoetxea, Fourier Analysis, Grad. Stud. Math. 29, Amer. Math. Soc., Providence, RI, 2001.

[9] J. García-Cuerva, Weighted $H^{p}$ spaces, Dissertationes Math. 162 (1979), 63 pp.

[10] J. García-Cuerva and J. L. Rubio de Francia, Weighted Norm Inequalities and Related Topics, North-Holland Math. Stud. 116, North-Holland, Amsterdam, 1985.

[11] L. Grafakos, On multilinear fractional integrals, Studia Math. 102 (1992), 49-56.

[12] -, Classical and Modern Fourier Analysis, Prentice-Hall, Englewood Cliffs, NJ, 2004.

[13] L. Grafakos and N. Kalton, Multilinear Calderón-Zygmund operators on Hardy spaces, Collect. Math. 52 (2001), 169-179.

[14] L. Grafakos and J. M. Martell, Extrapolation of weighted norm inequalities for multivariable operators and applications, J. Geom. Anal. 14 (2004), 19-46.

[15] L. Grafakos and R. H. Torres, Multilinear Calderón-Zygmund theory, Adv. Math. 165 (2002), 124-164.

[16] - - - Maximal operator and weighted norm inequalities for multilinear singular integrals, Indiana Univ. Math. J. 51 (2002), 1261-1276.

[17] - - -, On multilinear singular integrals of Calderón-Zygmund type, in: Proc. 6th International Conference on Harmonic Analysis and Partial Differential Equations (El Escorial, 2000), Publ. Mat. 2002, Vol. Extra, 57-91.

[18] L. I. Hedberg, On certain convolution inequalities, Proc. Amer. Math. Soc. 36 (1972), 505-510. 
[19] J. A. Hempel, G. R. Morris and N. S. Trudinger, On the sharpness of a limiting case of the Sobolev embedding theorem, Bull. Austral. Math. Soc. 3 (1970), 369-373.

[20] C. E. Kenig and E. M. Stein, Multilinear estimates and fractional integration, Math. Res. Lett. 6 (1999), 1-15.

[21] A. K. Lerner, S. Ombrosi, C. Pérez, R. H. Torres and R. Trujillo-González, New maximal functions and multiple weights for the multilinear Calderón-Zygmund theory, Adv. Math. 220 (2009), 1222-1264.

[22] Y. Lin and S. Z. Lu, Boundedness of multilinear singular integral operators on Hardy and Herz-type spaces, Hokkaido Math. J. 36 (2007), 585-613.

[23] K. Moen, Weighted inequalities for multilinear fractional integral operators, Collect. Math. 60 (2009), 213-238.

[24] R. S. Strichartz, A note on Trudinger's extension of Sobolev's inequalities, Indiana Univ. Math. J. 21 (1972), 841-842.

[25] J. O. Strömberg and A. Torchinsky, Weighted Hardy Spaces, Lecture Notes in Math. 1381, Springer, 1989.

[26] N. S. Trudinger, On imbeddings into Orlicz spaces and some applications, J. Math. Mech. 17 (1967), 473-483.

Wenjuan Li

School of Mathematical Sciences

Beijing Normal University

Laboratory of Mathematics

and Complex Systems

Ministry of Education

Beijing 100875, P.R. China

E-mail: facingworld@mail.bnu.edu.cn

Kôzô Yabuta

Research Center for Mathematical Sciences

Kwansei Gakuin University

Gakuen 2-1, Sanda 669-1337, Japan
Qingying Xue (corresponding author)

School of Mathematical Sciences

Beijing Normal University

Laboratory of Mathematics and Complex Systems Ministry of Education Beijing 100875, P.R. China and

Institute of Applied Physics and Computational Mathematics PO Box 8009, Beijing 100088, P.R. China

Current address:

Department of Mathematics

University of California Berkeley, CA 94720-3840, U.S.A.

E-mail: qyxue@bnu.edu.cn

Received November 11, 2009

Revised version April 27, 2010 